\title{
SECOND-ORDER TIME DEGENERATE PARABOLIC EQUATIONS
}

\author{
BY
}

\author{
MARGARET C. WAID(1)
}

ABSTRACT. We study the degenerate parabolic operator $L u=\sum_{i, j=1}^{n} a^{i j} u_{x_{j} x_{j}}+$ $\sum_{i=1}^{n} b^{i} u_{x_{i}}-c u_{t}+d u$ where the coefficients of $L$ are bounded, real-valued functions defined on a domain $D=\Omega \times(0, T] \subset R^{n+1}$. Classically, $c(x, t) \equiv 1$ or, equivalently, $c(x, t) \geq \eta>0$ for all $(x, t) \in \bar{D}$. We assume only that $c$ is nonnegative. We prove weak maximum principles and Harnack inequalities. Assume that $a^{i j}$ is constant, the coefficients of $L$ and $f$ and their derivatives with respect to time are uniformly Hölder continuous (exponent $\alpha$ ) in $\bar{D}, \bar{D}$ has sufficiently nice boundary, $c>0$ on the normal boundary of $D, \psi \in \bar{C}_{z+a}$, and $L \psi=f$ on $\partial B=\partial(\bar{D} \cap\{t=0\})$. Then there exists a unique solution $u$ of the first initialboundary value problem $L u=f, u=\psi$ on $\bar{B}+(\partial B \times[0, T])$; and, furthermore, $u \in \bar{C}_{2+a}$. All results require proofs that differ substantially from the classical ones.

1. Notation and preliminary remarks. In this paper we shall study the second-order nonuniformly parabolic operator

$$
L u=\sum_{i, j=1}^{n} a^{i j} u_{x_{i} x_{j}}+\sum_{i=1}^{n} b^{i} u_{x_{i}}-c u_{t}+d u .
$$

$u$ and all the coefficients of $L$ are real-valued functions defined for $(x, t)=$ $\left(x_{1}, \cdots, x_{n}, t\right)$ in an $(n+1)$-dimensional, bounded, convex domain $D$. We are using subscripts to denote differentiation.

We will assume that $L$ is parabolic; that is,

$$
\sum_{i, j=1}^{n} a^{i j} \xi_{i} \xi_{i} \geq \gamma|\xi|^{2}>0
$$

for any real vector $\xi \neq 0$. The coefficient $c$ will be assumed to be nonnegative. However, we will not make the usual assumption that $c$ is bounded away from zero. Since $c(x, t)=0$ for some $(x, t)$ in the closure of $D$ is permissible, $L$ may be a degenerate parabolic operator. We will make assumptions concerning the smoothness of the coefficients as needed.

Presented to the Society, July 6, 1971; received by the editors July 9, 1971 and, in revised form, December $1,1971$.

AMS 1970 subject classifications. Primary 35K15, 35K20, 35K99, 35B 45; Secondary 35J70, 35B30, 35M05.

Key words and phrases. Degenerate parabolic equations, nonuniformly parabolic operator, first initial-boundary value problem, maximum principles, Harnack inequality, a priori estimates, existence of a unique classical solution.

(1) These results are contained in the author's doctoral dissertation written under the direction of Professor Wayne T. Ford at Texas Tech University. 
We will prove the usual weak maximum principles for the operator (1.1). For the special case in which $u$ satisfies $L u=f$ with all the $a^{i j}$ constants, estimates are given for $\max \left|u_{t}\right|$ and $\max \left|u_{x_{i}}\right|$, in terms of their values on a portion of the boundary of $D$. Furthermore, these estimates are independent of the minimum of $c$ in $D$.

In addition, we will obtain Harnack inequalities, again independent of the minimum of $c$ in $D$, for a solution of the equation $L u=f$ where $L$ is as in (1.1). Classical proofs of both the maximum principles and the Harnack inequalities depend strongly on the assumption that $c(x, t) \geq \lambda>0$ for some constant $\lambda$ and for all $(x, t)$ in the closure of $D$. We have had to modify substantially the methods of proof, making some assumptions concerning the set of zeros of $c$ and, in some instances, requiring that $c_{t}$ be bounded.

The main result in this paper is a proof of the existence of a unique classical, or strong, solution to the first initial-boundary value problem. The method of proof employed involves perturbing the coefficient $c$ and obtaining a Cauchy sequence of solutions to the perturbed equations which converges in an appropriate Banach space to a solution of the first initial-boundary value problem. Showing that the sequence is Cauchy requires that we obta in somewhat sharper Schauder-type a priori boundary estimates than those of A. Friedman in [6]. That is, we must show exactly how Friedman's estimates depend on the minimum of $c$. Furthermore, our method of proof entails finding an a priori boundary estimate for $u_{t}$ which is independent of the minimum of $c$ in $D$.

We will use the same notation as A. Friedman [6, p. 40] in describing the domain $D$. As stated above, $D$ is a bounded, convex, $(n+1)$-dimensional domain in $R^{n+1}$, where $(x, t)=\left(x_{1}, \cdots, x_{n}, t\right)$ represents a variable point in $R^{n+1}$. For any set $A$, let $\bar{A}$ denote the closure of $A$. The union of two sets, $A$ and $B$, will be denoted by $A+B$. $\partial D$ denotes the boundary of $D ; \partial D=\bar{B}+B_{T}+S$, where $B$ is a domain in $R^{n} \times\{0\}, B_{T}(T>0)$ is a domain in $R^{n} \times\{T\}$, and $S$ is a manifold, not necessarily connected, in $R^{n} \times(0, T] . S+\bar{B}$ is generally called the parabolic, or normal, boundary of $D$.

Let $D_{\tau}=D \cap\left(R^{n} \times(0, \tau)\right), B_{\tau}=D \cap\left(R^{n} \times\{\tau\}\right)$, and $S_{\tau}=S \cap\left(R^{n} \times(0, \tau]\right)$. Assume that for each $\tau \in(0, T), B_{\tau}$ is a domain. Then, for every $(x, \tau)$ in $D$, $0<\tau<T$, if $S(x ; \tau)=D_{\tau}+B_{\tau}$, then $\overline{S(x, \tau)}-S(x, \tau)=\bar{B}+S_{\tau}$. A lso, assume that $D$ satisfies the following condition: there exists a simple continuous curve $a$ connecting $B$ to $B_{T}$ along which the $t$-coordinate is nondecreasing.

For an excellent, as well as comprehensive, discussion of the history and applications of the first initial-boundary value problem and other problems for degenerate quasilinear parabolic equations, the reader is referred to [13], which contains a series of lectures by Professor Olga Oleĭnik. Specific applications to the 
problem of flows through a porous media can be found in [2] and [4]. The numerical analysis for the case of one space variable has been studied by E. D. Williams [17].

2. Weak maximum principles. We now prove a sequence of weak maximum principles which are similar in nature to those proved by A. Friedman in [6]. In some instances, the proof by Friedman can be used with only minor modification. Most of the results, however, require considerable modification because they rely heavily on the fact that $c(x, t) \equiv 1$.

We assume, as does Friedman, that the coefficients of $L$, defined in (1.1), are continuous in $D$. Friedman remarks that the reader may observe that the majority of the theorems remain true if the coefficients are only bounded functions and the operators are assumed to be locally uniformly parabolic. The same remark applies for many of the theorems in this section.

Unless otherwise indicated, the operator $L$ is as defined in (1.1), where $L$ is parabolic (1.2) and $c$ is nonnegative. Assume also that $u$ is continuous on the closure of $D$ and that $u$ is of class $C^{2,1}(D)$.

Theorem 2.1. If $L u \leq 0(L u \geq 0), d<0$ in $D$, and $u \geq 0(u \leq 0)$ on $\Gamma=$ $\{(x, t) \in \partial D: t<T\}$, then $u \geq 0(u \leq 0)$ in the closure of $D_{T}=\{(x, t): t<T\}$.

Proof. Suppose that $u$ has a negative minimum at $\left(x^{0}, t^{0}\right)$ in $D_{T}$. Then,

$$
\sum_{i, j=1}^{n} a^{i j}\left(x^{0}, t^{0}\right) u_{x_{i} x_{j}}\left(x^{0}, t^{0}\right) \geq 0 \text {. }
$$

Hence

$$
\begin{aligned}
L u\left(x^{0}, t^{0}\right) & =\sum_{i, j=1}^{n} a^{i j}\left(x^{0}, t^{0}\right) u_{x_{i} x_{j}}\left(x^{0}, t^{0}\right)+d\left(x^{0}, t^{0}\right) u\left(x^{0}, t^{0}\right) \\
& \geq d\left(x^{0}, t^{0}\right) u\left(x^{0}, t^{0}\right)>0 .
\end{aligned}
$$

But, $L u\left(x^{0}, t^{0}\right) \leq 0$. This contradiction completes the proof.

Theorem 2.2. If $d$ is bounded above, $L u \leq 0(L u \geq 0)$ in $D$, and $u \geq 0(u \leq 0)$ on $\Gamma=\{(x, t) \in \partial D: t<T\}$, then $u \geq 0(u \leq 0)$ in the closure of $D_{T}=\{(x, t): t<T\}$.

Proof. Suppose that $q_{i}$ represents the breadth of $\bar{D}$ in the $x_{i}$-direction. In particular,

$$
\begin{aligned}
q_{i}=\min \{q>0: & \text { for some } \bar{x}_{i}, \bar{x}_{i}-q \leq x_{i} \leq \bar{x}_{i} \\
& \text { for all } \left.x \text { such that }\left(x_{1}, \cdots, x_{n}, t\right) \in \bar{D}\right\} .
\end{aligned}
$$

The minimum of this set exists since $\bar{D}$ is compact.

Without loss of generality, let us assume that $\bar{x}_{i}=q_{i}$; for if $\bar{x}_{i} \neq q_{i}$, we can perform a simple translation of the domain $D$ in the $x_{i}$-direction. We will use 
$0 \leq x_{1} \leq q_{1}, q_{1}>0$, in this proof.

Since the matrix $\left(a^{i j}\right)$ is positive definite and can be assumed symmetric, $a^{11} \geq \gamma_{1}>0$ for some constant $\gamma_{1}$. If $d(x, t)$ is identically the zero function, then $d(x, t)<\gamma_{1} / 4 q_{1}^{2}$. Suppose that $d(x, t)$ is positive for some $(x, t) \in D$. Let $K=\max \left\{d(x, t):(x, t) \in \bar{D}_{T}\right\}$.

If $\left(x_{1}, \cdots, x_{n}, t\right)$ is a point in $\bar{D}$, let $\left(z_{1}, x_{2}, \cdots, x_{n}, t\right)$ denote the projection toward zero of $(x, t)$ onto $S$ in the $x_{1}$-direction. Note that if $y_{1}$ is such that $z_{1}<y_{1}<x_{1}$, then $\left(y_{1}, x_{2}, \cdots, x_{n}, t\right) \in D$.

$\operatorname{Map}(x, t)$ to $\left(x^{\prime}, t\right)=\left(x_{1}^{\prime}, x_{2}, \cdots, x_{n}, t\right)$, where $x_{1}^{\prime}=z_{1}+\left(\gamma_{1} / 8 K q_{1}^{2}\right)^{1 / 2}\left(x_{1}-z_{1}\right)$ $=\left[1-\left(\gamma_{1} / 8 K q_{1}^{2}\right)^{1 / 2}\right] z_{1}+\left(\gamma_{1} / 8 K q_{1}^{2}\right)^{1 / 2} x_{1}$. Then $x_{1}=x_{1}^{\prime}\left(\gamma_{1} / 8 K q_{1}^{2}\right)^{-1 / 2}-$ $\left[\left(\gamma_{1} / 8 K q_{1}^{2}\right)^{-1 / 2}-1\right] z_{1}$. Let $v\left(x_{1}^{\prime}, x_{2}, \cdots, x_{n}, t\right)=u(x, t)$. We may assume that $\left(\gamma_{1} / 8 K q_{1}^{2}\right)^{1 / 2}<1$, for otherwise $d(x, t)<\gamma_{1} / 4 q_{1}^{2}$.

Let $D^{\prime}$ denote the domain obtained from $D$ by the above transformation. Since $\left(\gamma_{1} / 8 K q_{1}^{2}\right)^{1 / 2}<1, D^{\prime}$ is a subdomain of $D$. $L u \leq 0$ on $D$ and hence $L v \leq 0$ on $D^{\prime}$. $u \geq 0$ on $\Gamma=\{(x, t) \in \partial D: t<T\}$, so $v \geq 0$ on $\Gamma^{\prime}=\left\{(x, t) \in \partial D^{\prime}: t<T\right\}$. $q_{1}$ is mapped to $\left(\gamma_{1} / 8 K q_{1}^{2}\right)^{1 / 2} q_{1}=\left(\gamma_{1} / 8 K\right)^{1 / 2}=q_{1}^{\prime}$, and hence $q_{1}^{\prime}$ is the breadth of $D^{\prime}$ in the $x_{1}^{\prime}$-direction. A simple computation shows that $\gamma_{1} / 4\left(q_{1}^{\prime}\right)^{2}=2 K>d(x, t)$ for all $(x, t) \in D$. Since $D^{\prime}$ is a subdomain of $D$, it follows that $\gamma_{1} / 4\left(q_{1}^{\prime}\right)^{2}>$ $d\left(x_{1}^{\prime}, x_{2}, \cdots, x_{n}, t\right)$ for all $\left(x_{1}^{\prime}, x_{2}, \cdots, x_{n}, t\right)$ in $D^{\prime}$. If we show that the theorem holds for $v$ and the domain $D^{\prime}$, we will then have $v \geq 0$ in $\bar{D}_{T}^{\prime} \cdot\left(x_{1}^{\prime}, x_{2}, \ldots\right.$, $\left.x_{n}, t\right) \in \bar{D}_{T}^{\prime}$ implies that

$$
\left(z_{1}+\left(\gamma_{1} / 8 K q_{1}^{2}\right)^{1 / 2}\left(x_{1}^{\prime}-z_{1}\right), x_{2}, \ldots, x_{n}, t\right) \in \bar{D}_{T}^{\prime}
$$

Therefore,

$$
v\left(z_{1}+\left(y_{1} / 8 K q_{1}^{2}\right)^{1 / 2}\left(x_{1}^{\prime}-z_{1}\right), x_{2}, \cdots, x_{n}, t\right) \geq 0
$$

But

$$
v\left(z_{1}+\left(y_{1} / 8 K q_{1}^{2}\right)^{1 / 2}\left(x_{1}^{\prime}-z_{1}\right), x_{2}, \ldots, x_{n}, t\right)=u\left(x_{1}^{\prime}, x_{2}, \ldots, x_{n}, t\right)
$$

hence $u\left(x_{1}^{\prime}, x_{2}, \cdots, x_{n}, t\right) \geq 0$.

Consider whether $d(x, t) \leq \gamma_{1} / 4\left(q_{1}-q_{1}^{\prime}\right)^{2}$ on $D_{T}-\bar{D}_{T}^{\prime}$. If so, we can simply regard $D_{T}$ as the union of these two subdomains and prove the theorem for each subdomain separately. If not, we continue with this process until we have written $D_{T}$ as the union of a finite number $N$ of such domains on each of which $d(x, t)<\gamma_{1} / 4\left(q_{1}^{(n)}\right)^{2}, 1 \leq n \leq N . q_{1}^{(n)}$ represents the breadth of the $n$th domain. We are assured that this can be accomplished in a finite number of steps since $D$ is bounded.

We now return to the proof of the theorem for $v$ and the domain $D^{\prime}$. For convenience of notation, let us use $D$ for $D^{\prime}, u$ for $v, x_{1}$ for $x_{1}^{\prime}$, and $q_{1}$ for $q_{1}^{\prime}$. Let $v=u\left(e^{\epsilon x_{1}}+\alpha e^{-\epsilon x_{1}}\right)=u \cdot g\left(x_{1}\right)$ where $\epsilon, \alpha>0$ are chosen so that 
$1-2\left(e^{\epsilon x_{1}}-\alpha e^{-\epsilon x_{1}}\right)^{2}\left(g\left(x_{1}\right)\right)^{-2}>0 . \epsilon, a$ can always be so chosen since

$$
\begin{aligned}
1-2\left(e^{\epsilon x_{1}}-\alpha e^{-\epsilon x_{1}}\right)^{2}\left(g\left(x_{1}\right)\right)^{-2} \\
\quad=\left(-e^{2 \epsilon x_{1}}+6 a-\alpha^{2} e^{-2 \epsilon x_{1}}\right)\left(g\left(x_{1}\right)\right)^{-2} \\
\geq\left(-e^{2 \epsilon q_{1}}+6 a-\alpha^{2}\right)\left(g\left(x_{1}\right)\right)^{-2}=j(\alpha)\left(g\left(x_{1}\right)\right)^{-2} \\
\geq j(\alpha) / 16
\end{aligned}
$$

for $a, \epsilon$ such that $j(\alpha) \geq 0, j^{\prime}(\alpha)=6-2 \alpha, j^{\prime \prime}(\alpha)=-2$. Hence, $j$ has a maximum at $a=3$. Let $\epsilon=\left(2 q_{1}\right)^{-1}$. Then $j(3)=9-e \geq 1$. Hence $v=u\left(e^{x_{1} / 2 q_{1}}+3 e^{-x_{1} / 2 q_{1}}\right)$ $=u \cdot g\left(x_{1}\right)$ can be chosen as the specified transformation.

Some useful observations concerning $g$ are

$$
g^{\prime}\left(x_{1}\right)=\left(2 q_{1}\right)^{-1}\left(e^{x_{1} / 2 q_{1}}-3 e^{-x_{1} / 2 q_{1}}\right)<0
$$

and

$$
1<g\left(x_{1}\right) \leq 4
$$

$v \in C(\bar{D})$ and $C^{2,1}(D)$ since $u \in C(\bar{D})$ and $C^{2,1}(D)$.

A routine computation gives

$$
\begin{aligned}
L v= & g\left(x_{1}\right) L u \\
& +a^{11}\left[2 g^{\prime}\left(x_{1}\right)\left(g\left(x_{1}\right)\right)^{-1}\left(v_{x_{1}}-g^{\prime}\left(x_{1}\right)\left(g\left(x_{1}\right)\right)^{-1} v\right)+\left(4 q_{1}^{2}\right)^{-1} v\right] \\
& +\sum_{j=2}^{n} a^{1 j} g^{\prime}\left(x_{1}\right)\left(g\left(x_{1}\right)\right)^{-1} v_{x_{j}} \\
& +\sum_{i=2}^{n} a^{i 1} g^{\prime}\left(x_{1}\right)\left(g\left(x_{1}\right)\right)^{-1} v_{x_{i}}+b^{1} g^{\prime}\left(x_{1}\right)\left(g\left(x_{1}\right)\right)^{-1} v .
\end{aligned}
$$

Let

$$
\begin{aligned}
M v= & L v-\sum_{j=2}^{n} a^{1 j} g^{\prime}\left(x_{1}\right)\left(g\left(x_{1}\right)\right)^{-1} v_{x_{j}}-a^{11}(2) g^{\prime}\left(x_{1}\right)\left(g\left(x_{1}\right)\right)^{-1} v_{x_{1}} \\
& -\sum_{i=2}^{n} a^{i 1(2)} g^{\prime}\left(x_{1}\right)\left(g\left(x_{1}\right)\right)^{-1} v_{x_{i}}-b^{1}(2) g^{\prime}\left(x_{1}\right)\left(g\left(x_{1}\right)\right)^{-1} v \\
& =g\left(x_{1}\right) L u+a^{11}\left(4 q_{1}^{2}\right)^{-1}\left[1-2\left(e^{x_{1} / 2 q_{1}}-3 e^{\left.\left.-x_{1} / 2 q_{1}\right)^{2}\left(g\left(x_{1}\right)\right)^{-2}\right] v}\right.\right. \\
& \leq a^{11}\left(4 q_{1}^{2}\right)^{-1}\left[1-2\left(e^{x_{1} / 2 q_{1}}-3 e^{-x_{1} / 2 q_{1}}\right)^{2}\left(g\left(x_{1}\right)\right)^{-2}\right] v .
\end{aligned}
$$


Therefore,

$$
\begin{aligned}
N v & =\left(M-a^{11}\left(4 q_{1}^{2}\right)^{-1}\left[1-2\left(e^{x_{1} / 2 q_{1}}-3 e^{\left.\left.\left.-x_{1} / 2 q_{1}\right)^{2}\left(g\left(x_{1}\right)\right)^{-2}\right]\right) v}\right.\right.\right. \\
& \leq d(x, t)-a^{11}(x, t) / 4 q_{1}^{2}<0 .
\end{aligned}
$$

We have already that $v \geq 0$ on $\Gamma$. By Theorem 2.1, $v \geq 0$ on $\bar{D}_{T} \cdot g\left(x_{1}\right)>0$ and $v \geq 0$ imply $u \geq 0$ on $\bar{D}_{T}$. This completes the proof of the theorem.

The following are corollaries to the above maximum principle. If the proof is no different from the case $c(x, t) \equiv 1$, it is omitted.

If $u$ is a bounded function on a subset $S$ of $R^{n}$, define $M(u ; S)=\sup \{u(x, t):(x, t) \in S\} \quad$ and $\quad m(u ; S)=\inf \{u(x, t):(x, t) \in S\}$.

Corollary 2.3. Lu>0 and $d \equiv 0$ in $D$ imply $u$ bas no relative maximum in $D$.

Corollary 2.4. If $L u=0$ and $d$ is bounded above, then $M\left(|u| ; \bar{D}_{T}\right) \leq$ $M\left(|u| ; \bar{B}+S_{T}\right)$.

Corollary 2.5. If $d(x, t) \leq 0, a^{11} \lambda^{2}+b^{1} \lambda \geq 1$ for some $\lambda>0$, and if $L u=f$ in $D$, then

$$
M\left(|u| ; \bar{D}_{T}\right) \leq M\left(|u| ; \bar{B}+S_{T}\right)+\left(e^{\lambda q_{1}}-1\right) M(|f| ; \bar{D}) .
$$

Corollary 2.6. If $d<a^{11} / 4 q_{1}^{2}, a^{11} \lambda^{2}+b^{1} \lambda \geq 1$ for some $\lambda>0$, and if $L u=f$ in $D$, then

$$
M\left(|u| ; \bar{D}_{T}\right) \leq 4\left[M\left(|u| ; \bar{B}+S_{T}\right)+\left(e^{\lambda q_{1}}-1\right) M\left(|f| ; \bar{D}_{T}\right)\right] .
$$

Proof. Applying Corollary 2.5 to $v=u \cdot g\left(x_{1}\right)$, where $g\left(x_{1}\right)$ is as in the proof of Theorem 2.2, it follows that

$$
M\left(|v| ; \bar{D}_{T}\right) \leq M\left(|v| ; \bar{B}+S_{T}\right)+\left(e^{\lambda q}-1\right) M\left(\left|g\left(x_{1}\right) f\right| ; \bar{D}_{T}\right) .
$$

$1 \leq g\left(x_{1}\right) \leq 4$ guarantees that

$$
M\left(|u| ; \bar{D}_{T}\right) \leq M\left(|v| ; \bar{D}_{T}\right) \leq 4 M\left(|u| ; \bar{B}+S_{T}\right)+\left(e^{\lambda q_{1}}-1\right) M\left(|f| ; \bar{D}_{T}\right) .
$$

Corollary 2.7. If $d$ is bounded above by $K>0, a^{1{ }^{1}} \lambda^{2}+b^{1} \lambda \geq 1$ for some $\lambda>0$, and if $L u=f$ in $D$, then

$$
M\left(|u| ; \bar{D}_{T}\right) \leq 4\left[M\left(|u| ; \bar{B}+S_{T}\right)+\left(\exp \left\{\lambda\left(\gamma_{1} / 8 K\right)^{1 / 2}\right\}-1\right) M\left(|f| ; \bar{D}_{T}\right)\right] .
$$

Proof. Map $x_{1}$ to $x_{1}^{\prime}=\left(\gamma_{1} / 8 K q_{1}^{2}\right)^{1 / 2} x_{1}$, and let $v\left(x^{\prime}, t\right)=v\left(x_{1}^{\prime}, x_{2}, \cdots, x_{n}, t\right)=$ $u(x, t)$. Then $q_{1}$ is mapped to $q_{1}^{\prime}=\left(\gamma_{1} / 8 K q_{1}^{2}\right)^{1 / 2} q_{1}=\gamma_{1} / 8 K$ and $\gamma_{1} / 4\left(q_{1}^{\prime}\right)^{2}=$ $2 K>d$. Let $D^{\prime}$ be the image of $D$ under the above map.

By Corollary 2.6,

$$
M\left(|\nu| ; \bar{D}_{T}^{\prime}\right) \leq 4\left[M\left(|\nu| ; \bar{B}^{\prime}+S_{T}^{\prime}\right)+\left(\exp \left\{\lambda\left(q_{1}^{\prime}\right)^{2}\right\}-1\right) M\left(\left|\hat{f}\left(x^{\prime}, t\right)\right| ; \bar{D}_{T}^{\prime}\right)\right]
$$


where $\hat{f}\left(x^{\prime}, t\right)=f(x, t)$, and the desired inequality follows.

Theorem 2.8. Assume that $a^{i j}$ is constant for each $i, j$, that all coefficients of $L$ are of class $C^{1,1}(\bar{D}), u \in C(\bar{D}), u \in C^{3}(D)$, and $a^{1{ }^{1}} \lambda^{2}+b^{1} \lambda \geq 1$ for some $\lambda>0$. Then there is a constant $C$ which depends only on $\lambda, M(|\alpha| ; \bar{D})$, where $a \in\left\{b_{t}, c_{t}, d_{x_{i}}, b_{x_{i}}, c_{x_{i}}\right\}$, such that

$$
\begin{aligned}
M\left(\left|u_{t}\right| ; \bar{D}_{T}\right) \leq C\left[M \left(\left|u_{t}\right| ; \bar{B}+\right.\right. & \left.S_{T}\right)+\sum_{i=1}^{n} M\left(\left|u_{x_{i}}\right| ; \bar{B}+S_{T}\right)+M\left(|u| ; \bar{B}+S_{T}\right) \\
& \left.+M\left(\left|f_{t}\right| ; \bar{D}_{T}\right)+\sum_{i=1}^{n} M\left(\left|f_{x_{i}}\right| ; \bar{D}_{T}\right)+M\left(|f| ; \bar{D}_{T}\right)\right] .
\end{aligned}
$$

Proof. We will indicate the proof for the case of one space variable, since in doing so we lose only a good deal of tedium and virtually none of the technique. Suppose that $K>0$ is an upper bound for $d-c_{t}$ and $d+b_{x}$.

Consider $L u=a u_{x x}+b u_{x}-c u_{t}+d u=f$. If we differentiate both sides of $L u=f$ with respect to $t$, it follows that $\left(L-c_{t}\right) u_{t}=f_{t}-b_{t} u_{x}-d_{t} u$. Corollary 2.7 implies that

$$
\begin{aligned}
1 / 4 M\left(\left|u_{t}\right| ; \bar{D}_{T}\right) \leq & M\left(\left|u_{t}\right| ; \bar{B}+S_{T}\right)+\left(\exp \left\{\lambda(a / 8 K)^{1 / 2}\right\}-1\right) \\
& \cdot\left[M\left(\left|f_{t}\right| ; \bar{D}_{T}\right)+M\left(\left|b_{t}\right|\left|u_{x}\right| ; \bar{D}_{T}\right)+M\left(\left|d_{t}\right||u| ; \bar{D}_{T}\right)\right] .
\end{aligned}
$$

Differentiating, instead, with respect to $x$ yields $\left(L+b_{x}\right) u_{x}=f_{x}+c_{x} u_{t}$ $-d_{x} u$. Apply Corollary 2.7 again to see that

$$
\begin{aligned}
1 / 4 M\left(\left|u_{x}\right| ; \bar{D}_{T}\right) \leq & M\left(\left|u_{x}\right| ; \bar{B}+S_{T}\right)+\left(\exp \left\{\lambda(a / Q K)^{1 / 2}\right\}-1\right) \\
& \cdot\left[M\left(\left|f_{x}\right| ; \bar{D}_{T}\right)+M\left(\left|c_{x}\right|\left|u_{t}\right| ; \bar{D}_{T}\right)+M\left(\left|d_{x}\right||u| ; \bar{D}_{T}\right)\right] .
\end{aligned}
$$

Substitute (2.4) into (2.3), so that

$$
\begin{aligned}
& \frac{1 / 4 M\left(\left|u_{t}\right| ; \bar{D}_{T}\right) \leq M}{}\left(\left|u_{t}\right| ; \bar{B}+S_{T}\right) \\
&+\left(\exp \left\{\lambda(a / 8 K)^{1 / 2}\right\}-1\right) \\
& \cdot\left[M\left(\left|f_{t}\right| ; \bar{D}_{T}\right)+4 M\left(\left|b_{t}\right| ; \bar{D}_{T}\right) M\left(\left|u_{x}\right| ; \bar{B}+S_{T}\right)\right. \\
&+\left(\exp \left\{\lambda(a / 8 K)^{1 / 2}\right\}-1\right) \\
& \cdot\left(M\left(\left|f_{x}\right| ; \bar{D}_{T}\right)+M\left(\left|c_{x}\right|\left|u_{t}\right| ; \bar{D}_{T}\right)+M\left(\left|d_{x}\right||u| ; \bar{D}_{T}\right)\right) \\
&\left.+M\left(\left|d_{x}\right||u| ; \bar{D}_{T}\right)\right] .
\end{aligned}
$$

If $c_{x} \equiv 0$, we have the desired inequality. If $c_{x} \not \equiv 0$, then let $K_{1}=$ $M\left(\left|c_{x}\right| ; \bar{D}_{T}\right)>0$. Make a change in the time scale so that $M\left(\left|b_{t}\right| ; \bar{D}_{T}\right)<$ $\left[32 K_{1}\left(\exp \left\{\lambda(a / 8 K)^{1 / 2}\right\}-1\right)^{2}\right]^{-1}$. Then 


$$
1 / 4 M\left(\left|u_{t}\right| ; \bar{D}_{T}\right) \leq C\left[M\left(\left|u_{t}\right| ; \bar{B}+S_{T}\right)+M\left(\left|u_{x}\right| ; \bar{B}+S_{T}\right)+M\left(|u| ; \bar{B}+S_{T}\right)\right.
$$

$$
\left.+M\left(\left|f_{t}\right| ; \bar{D}_{T}\right)+M\left(\left|f_{x}\right| ; \bar{D}_{T}\right)+M\left(|f| ; \bar{D}_{T}\right)+(1 / 8) M\left(\left|u_{t}\right| ; \bar{D}_{T}\right)\right] \text {. }
$$

Multiply both sides of (2.6) by 8 , and then subtract $M\left(\left|u_{t}\right| ; \bar{D}_{T}\right)$ from both sides. This completes the proof of the theorem.

Corollary 2.9. Make the same assumptions regarding $u$ and the coefficients of $L$ as in Theorem 2.8. Then there is a constant $C$ which depends only on $\lambda$, $M(|\alpha| ; \bar{D})$, where $a \in\left\{b_{t}, c_{t}, d_{x_{i}}, b_{x_{i}}, c_{x_{i}}\right\}$ such that

$$
\begin{aligned}
M\left(\left|u_{x_{i}}\right| ; \bar{D}_{T}\right) \leq C\left[M\left(\left|u_{t}\right| ; \bar{B}+S_{T}\right)\right. & +\sum_{i=1}^{n} M\left(\left|u_{x_{i}}\right| ; \bar{B}+S_{T}\right)+M\left(|u| ; \bar{B}+S_{T}\right) \\
& \left.+M\left(\left|f_{t}\right| ; \bar{D}_{T}\right)+\sum_{i=1}^{n} M\left(\left|f_{x_{i}}\right| ; \bar{D}_{T}\right)+M\left(|f| ; \bar{D}_{T}\right)\right] .
\end{aligned}
$$

Proof. Use the estimate obtained for $M\left(\left|u_{t}\right| ; \bar{D}_{T}\right)$ in Theorem 2.8 in (2.5).

3. Harnack inequalities. We now derive a Harnack inequality which is similar to the Harnack inequality for nonnegative harmonic functions. For elliptic equations, the inequality gives a bound for the maximum of $u$ over an interior subset of the domain of definition in terms of a minimum of $u$ on the same subset. For parabolic equations it is necessary that we consider two subsets separated by a nonempty time interval. J. Moser in [12] provides an excellent discussion indicating a physical interpretation of this phenomenon as well as giving an example to show why it is a necessary condition.

The proof we shall give is similar in nature to the proof for the special case $u_{t}=\sum_{i, j=1}^{n}\left(a^{i j} u_{x_{i}}\right)_{x_{j}}$ given by Moser in [12]. It is, in fact, a modification of the case $c \equiv 1$, given by D. G. Aronson and J. Serrin in [3]. In case a portion of the argument is identical to that of Aronson and Serrin, it will be omitted or briefly outlined.

In order to apply this method to the equation $L u=f$, where $L$ is the operator (1.1), we note that $L u=f$ can be expressed in the form

$$
\sum_{i, j=1}^{n}\left\{a^{i j}(x, t) u_{x_{i}}\right\}_{x_{j}}+\sum_{i=1}^{n}\left(b^{i}-\sum_{j=1}^{n} a_{x_{j}}^{i j}\right) u_{x_{i}}+(1-c) u_{t}+d u-f=u_{t} \text {. }
$$

For $(x, t)=\left(x_{1}, x_{2}, \cdots, x_{n}, t\right) \in D=\Omega \times(0, T]$, (3.1) can be written as

$$
\operatorname{div} A\left(x, t, u_{x}\right)+B\left(x, t, u, u_{x}, u_{t}\right)=u_{t}
$$

where $A=\left(A_{1}, A_{2}, \cdots, A_{n}\right)$ is a given vector function of $\left(x, t, u_{x}\right), B$ is a given scalar function of $\left(x, t, u, u_{x}, u_{t}\right)$, and $u_{x}=\left(u_{x_{1}}, u_{x_{2}}, \cdots, u_{x_{n}}\right)$ denotes 
the spatial gradient of the dependent variable $u=u(x, t) . \operatorname{div} A$ is the divergence of $A\left(x, t, u_{x}(x, t)\right)$ with respect to the variables $\left(x_{1}, \cdots, x_{n}\right)$. The structure of (3.2) will be determined by $A(x, t, p)$ and $B(x, t, u, p, q)$.

We now define the classes $L^{p, q}(D)$. Let $u=u(x, t)$ be a measurable function defined on $D$. We say that $u \in L^{p, q}(D)$ if

$$
\left.\|u\|_{p, q}=\left\{\int_{0}^{T}\left(\int_{\Omega}|u|^{p} d x\right)^{q / p} d t\right\}\right\}^{1 / q}<\infty
$$

where $1 \leq p, q \in R$. $p$ or $q$ can be $\infty$ if the $L^{\infty}$ norm is used.

The structure inequalities which $A$ and $B$ satisfy are

$$
\begin{aligned}
p \cdot A(x, t, p) & \geq \gamma|p|^{2}, \\
|B(x, t, u, p, q)| & \leq M|p|+|1-c||q|+|d||u|+|f|, \\
|A(x, t, p)| & \leq \mu|p|,
\end{aligned}
$$

where $\gamma, \mu, M$ are positive constants, $M \geq\left|b^{i}-\sum_{j=1}^{n} a_{x_{j}}^{i j}\right|$ for all $i, j$, and the coefficients $|1-c|,|d|,|f|$ are each contained in some space $L^{p, q}(D)$, where $p$ and $q$ are nonnegative real numbers (possibly different from coefficient to coefficient) such that

$$
p>1 \text { and } n(2 p)^{-1}+(q)^{-1}<1 .
$$

The norms of $|1-c|,|d|,|f|$ in their respective spaces will be denoted by $\|1-c\|,\|d\|,\|f\|$.

Suppose that a function $v=v(x, t)$ is locally integrable in $D . v$ is said to be strongly differentiable with respect to $x$ if there exists a locally integrable vector function $v_{x}(x, t)$ such that $\iint_{D} v \phi_{x} d x d t=-\iint_{D} \phi v_{x} d x d t$ for all functions $\phi \in C_{0}^{1}(D)=\left\{\phi \in C^{1}(D): \phi\right.$ has compact support in $\left.D\right\}$. Similarly, one defines strong differentiability with respect to $t$.

If $u$ is a function which is locally of class $L^{2, \infty}$ in $\Omega$ and has a strong derivative $u_{x}$, which is locally of class $L^{2,2}$ in $\Omega$, we will say that $u$ is a weak solution of (3.2) if

$$
\iint_{D}\left(-u \phi_{t}+\phi_{x} A\left(x, t, u_{x}\right)\right) d x d t=\iint_{D} \phi B\left(x, t, u, u_{x}, u_{t}\right) d x d t
$$

for any $\phi \in C_{0}^{1}(D)$.

If $u$ is a strong solution of (3.2), then $u$ not only satisfies (3.6), it satisfies

$$
\iint_{D}\left(\phi u_{t}+\phi_{x} A\left(x, t, u_{x}\right)\right) d x d t=\iint_{D} \phi B\left(x, t, u, u_{x}, u_{t}\right) d x d t
$$

for any $\phi \in C_{0}^{1}(D)$.

Recall that $|1-c|,|d|$, and $|f|$ are each contained in some $L^{p, q}(D)$, where $p$ and $q$ satisfy (3.5).

Then there exists a positive constant $\theta$ such that 


$$
p \geq(1-\theta)^{-1} \text { and } n(2 p)^{-1}+(q)^{-1} \leq 1-\theta .
$$

One constant $\theta$ works for all of the three possible pairs of $(p, q)$.

We say that a constant depends on the structure of (3.2) if it is determined by the constants $\gamma, \mu, M,\|d\|,\|1-c\|,\|f\|, \theta$, and $n$ (and is uniformly bounded whenever these quantities are).

Let $(\bar{x}, \bar{t})$ be a fixed point in $D$. Denote by $R(\rho)$ the open cube in $R^{n}$ of edge length $\rho>0$ centered at $\bar{x}$, and define $D(\rho)=R(\rho) \times\left(\bar{t}-\rho^{2}, \bar{t}\right)$. The symbol $\|\cdot\|_{p, q, \rho}$ will denote the $L^{p, q}$ norm of a function over the cylinder $D(\rho)$. Let $D^{*}(\rho)=R(\rho) \times\left(\bar{t}-8 \rho^{2}, \bar{t}-7 \rho^{2}\right)$, so that $D^{*}(\rho)$ is simply the cylinder $D(\rho)$ translated toward zero in the $t$-direction a distance $7 \rho^{2}$.

In the theorem of Harnack type to follow, we will assume that $u$ is bounded and satisfies $L u=f$, where $L$ is defined as in (1.1), and the functions $u, a^{i j}$, $b^{i}, c, d, f$ satisfy $|u|,\left|a^{i j}\right|,\left|b^{i}\right|,|1-c|,|d|,|f|,\left|b^{i}-\sum_{j=1}^{n} a_{x_{j}}^{i j}\right|<K$. The structure inequalities (3.4) are then of the form

$$
\begin{aligned}
p \cdot A(x, t, p) & \geq \gamma|p|^{2}, \\
|B(x, t, u, p, q)| & \leq K(|p|+|q|+|u|+1), \\
|A(x, t, p)| & \leq K|p| .
\end{aligned}
$$

The collection of conditions listed in this paragraph shall be referred to as $(*)$.

Theorem 3.1. Suppose that $u$ is a bounded nonnegative classical solution of (3.2) in $D$ and that the conditions (*), described immediately preceding this theorem, are satisfied. If $D(3 \rho) \subset D$, then

$$
\underset{D^{*}(\rho)}{\operatorname{ess} \max } u \leq C\left(\begin{array}{l}
\text { ess min } \\
D(\rho)
\end{array}\left(u+\rho^{\theta} k\right)\right)
$$

where $C$ is a positive constant depending only on $\rho$ and the structure of (3.2), $\theta$ is defined in (3.8), and $k=\|f\|+\|1-c\|$. Furtbermore, $C$ is independent of $\|f\|,\|1-c\|$.

Proof. Aronson and Serrin give a normalization argument in [3, p. 97] to show that there is no loss of generality in assuming that $\rho=1 / 3$ and that $D=$ $D(3 \rho)=R(1) \times(0,1)$.

For arbitrary $\epsilon>0$, define $\bar{u}=u+k+\epsilon$. We will show that

$$
\begin{aligned}
& \text { ess } \max \\
& D^{*}(1 / 3)
\end{aligned} \leq C\left(\begin{array}{l}
\text { ess min } \bar{u} \\
D(1 / 3)
\end{array}\right)
$$

which is sufficient to prove the theorem.

Let $G(u)=\bar{u}^{\beta}$, where $\beta$ is a fixed nonzero real number. If $H(u)$ is defined by 


$$
H(u)= \begin{cases}(\beta+1)^{-1} \bar{u}^{\beta+1}, & \text { if } \beta \neq-1 \\ \log \bar{u}, & \text { if } \beta=-1\end{cases}
$$

then $H^{\prime}(u)=G(u)$.

Let $\chi\left(t, \tau_{1}, \tau_{2}\right)$ denote the characteristic function of the open interval $\left(\tau_{1}, \tau_{2}\right)$, with $0<\tau_{1}<\tau_{2}<T$; and let $\phi(x, t)=\eta^{2} G(u) \chi\left(t, \tau_{1}, \tau_{2}\right)$, where $\eta=$ $\eta(x, t)$ is a piecewise-smooth nonnegative function vanishing in a neighborhood of the parabolic boundary. $\bar{u}$ is bounded, and hence $\phi$ is admissible as a test function in (3.7).

Note that

$$
\phi_{x}= \begin{cases}\eta^{2} G^{\prime}(u) u_{x}+2 \eta \eta_{x} G(u), & t \in\left(\tau_{1}, \tau_{2}\right), \\ 0, & t \notin\left(\tau_{1}, \tau_{2}\right) .\end{cases}
$$

Also, $(H(u))_{t}=G(u) u_{t}$ and $\eta^{2} G(u) u_{t}=\left(\eta^{2} H(u)\right)_{t}-2 \eta \eta_{t} H(u)$. Then

(3.11) $\iint_{D} \phi u_{t} d x d t=\int_{\tau_{1}}^{\tau_{2}} \int_{\Omega} \eta^{2} G(u) u_{t} d x d t=\left.\int_{\Omega}\left(\eta^{2} H\right)\right|_{t=\tau_{1}} ^{t=\tau_{2}}-\int_{\tau_{1}}^{\tau_{2}} \int_{\Omega} 2 \eta \eta_{t} H d x d t$

Thus, if $\phi=\eta^{2} G(u) \chi\left(t, \tau_{1}, \tau_{2}\right)$ is used as a test function in (3.7), (3.11) implies

$$
\left.\int_{\Omega} \eta^{2} H(u)\right|_{t=\tau_{1}} ^{t=\tau_{2}} d x+\iint_{D}\left(\phi_{x} A-\phi B\right) d x d t=\int_{\tau_{1}}^{\tau_{2}} \int_{\Omega} 2 \eta \eta_{t} H(u) d x d t .
$$

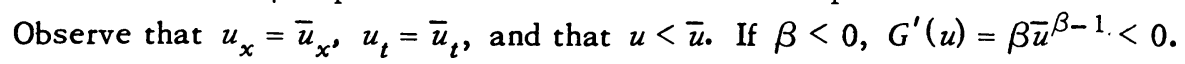
Thus,

$$
\begin{aligned}
& (\operatorname{sgn} \beta)\left(\phi_{x} A-\phi B\right) \\
& \quad \geq \eta^{2} \gamma\left|G^{\prime}\right|\left|\bar{u}_{x}\right|^{2}-2 \eta\left|\eta_{x}\right| G\left|\bar{u}_{x}\right|-\eta^{2} G K\left(\left|u_{x}\right|+\left|u_{t}\right|+|u|+1\right) .
\end{aligned}
$$

A simple computation shows that $G^{2}=\bar{u}^{\beta+1} G^{\prime} \beta^{-1}$. The next inequality is trivial to prove:

Similarly,

$$
2 \eta\left|\eta_{x}\right| G\left|\bar{u}_{x}\right| \leq|\beta| \eta^{2} G^{2}\left|u_{x}\right|^{2}\left(4 \bar{u}^{\beta+1}\right)^{-1}+4\left|\eta_{x}\right|^{2} \bar{u}^{\beta+1}|\beta|^{-1}
$$

$$
K G\left|\bar{u}_{x}\right| \leq|\beta| G^{\prime}\left|\bar{u}_{x}\right|^{2}(4 \beta)^{-1}+K^{2} \bar{u}^{\beta+1}|\beta|^{-1} .
$$

$G=\bar{u}^{\beta}$ and $k \leq \bar{u}$ imply

$$
K G(\bar{u}+1) \leq K\left(1+k^{-1}\right) \bar{u}^{\beta+1} .
$$

Combining inequalities $(3.13)-(3.16)$ yields 
$(\operatorname{sgn} \beta)\left(\phi_{x} A-\phi B\right) \geq \eta^{2} \gamma\left|G^{\prime}\right|\left|\bar{u}_{x}\right|^{2}-|\beta| \eta^{2} G^{\prime}\left|\bar{u}_{x}\right|^{2}(4 \beta)^{-1}$

$$
\begin{aligned}
& -4\left|\eta_{x}\right|^{2} \bar{u}^{\beta+1}|\beta|^{-1}-\eta^{2}|\beta| G^{\prime}\left|\bar{u}_{x}\right|^{2}(4 \beta)^{-1} \\
& -\eta^{2} K^{2} \bar{u}^{\beta+1}|\beta|^{-1}-K G\left|\bar{u}_{t}\right|-\eta^{2} K\left(1+k^{-1}\right) \bar{u}^{\beta+1} \\
= & 1 / 2|\beta| \gamma \eta^{2} \bar{u}^{\beta-1}\left|\bar{u}_{x}\right|^{2}-\eta^{2} K G\left|\bar{u}_{t}\right| \\
& -\bar{u}^{\beta+1}\left(4\left|\eta_{x}\right|^{2}|\beta|^{-1}+\eta^{2} K^{2}|\beta|^{-1}+\eta^{2} K\left(1-k^{-1}\right)\right)
\end{aligned}
$$

Hence,

$$
\eta^{2} K G\left|u_{t}\right|+\left(F \eta^{2}+E\left|\eta_{x}\right|^{2}\right) \bar{u}^{\beta+1}+(\operatorname{sgn} \beta)\left(\phi_{x} A-\phi B\right) \geq 1 / 2|\beta| \gamma \eta^{2} \bar{u}^{\beta-1}\left|\bar{u}_{x}\right|^{2},
$$

where $F=K^{2}|\beta|^{-1}+K\left(1-k^{-1}\right)$ and $E=4|\beta|^{-1}$.

It follows that

$$
\begin{aligned}
(\operatorname{sgn} \beta)\left\{\int_{\Omega} \eta^{2} H\right. & \left.\left.\right|_{t=\tau_{1}} ^{t=\tau_{2}} d x+(\beta / 2) \iint_{D} \eta^{2} \bar{u}^{\beta-1}\left|\bar{u}_{x}\right|^{2} d x d t\right\} \\
\leq & \iint_{D} \eta^{2} K G\left|\bar{u}_{t}\right| d x d t+\iint_{D} \sqrt{u}^{\beta+1} d x d t+2 \iint_{D} \eta\left|\eta_{t}\right||H| d x d t,
\end{aligned}
$$

where $J=F \eta^{2}+E\left|\eta_{x}\right|^{2}$. But, if $\beta \neq-1$,

$$
\begin{aligned}
\iint_{D} \eta^{2} K G\left|\bar{u}_{t}\right| d x d t & =\iint_{D} \eta^{2} K \bar{u}^{\beta}\left|\bar{u}_{t}\right| d x d t \\
& =\left(\operatorname{sgn} \bar{u}_{t}\right) \iint_{D} \eta^{2} K(\beta+1)^{-1}\left(\bar{u}^{\beta+1}\right)_{t} d x d t \\
& =-\left(\operatorname{sgn} \bar{u}_{t}\right)(\beta+1)^{-1} \iint_{D}\left(\eta^{2} K\right)_{t} \bar{u}^{\beta+1} d x d t \\
& =-\left(\operatorname{sgn} \bar{u}_{t}\right)(\beta+1)^{-1} \iint_{D} 2 \eta \eta_{t} K \bar{u}^{\beta+1} d x d t, \text { since } \eta \in C_{0}^{1}(D) .
\end{aligned}
$$

Now

$$
-\left(\operatorname{sgn} \bar{u}_{t}\right)(\beta+1)^{-1} \iint_{D} 2 \eta \eta_{t} K \bar{u}^{\beta+1} d x d t \leq|\beta+1|^{-1} \iint_{D} 2 \eta\left|\eta_{t}\right| K \bar{u}^{\beta+1} .
$$

We have thus proved the basic inequality

$$
\begin{aligned}
(\operatorname{sgn} \beta)\left\{\left.\int_{\Omega} \eta^{2} H\left|\begin{array}{l}
t \\
t
\end{array}=\tau_{2} d x+(\beta / 2) \iint_{D} \eta^{2} \bar{u}^{\beta-1}\right| \bar{u}_{x}\right|^{2} d x d t\right\} \\
\leq \iint_{D} J \bar{u}^{\beta+1} d x d t+(2+2 K)|\beta+1|^{-1} \iint_{D} \eta\left|\eta_{t}\right| \bar{u}^{\beta+1} d x d t,
\end{aligned}
$$

where $J=F \eta^{2}+E\left|\eta_{x}\right|^{2}$, which holds for $\beta \neq-1$.

Let us now consider the case $\beta=-1$. Recall that (3.17) holds for all $\beta$. If $\beta=-1$, then $H=\log \bar{u}, G=\bar{u}^{-1}$. Then (3.17) becomes 


$$
\begin{aligned}
(-1)\left\{\left.\int_{\Omega} \eta^{2} \log \bar{u}\left|\begin{array}{l}
t \\
t
\end{array}=\tau_{1} d x-\frac{1}{2} \iint_{D} \eta^{2} \bar{u}^{-2}\right| \bar{u}_{x}\right|^{2} d x d t\right\} \\
\leq \iint_{D} J d x d t+2 \iint_{D} \eta\left|\eta_{t}\right| \log \bar{u} d x d t+\iint_{D} \eta^{2} K \bar{u}^{-1}\left|\bar{u}_{t}\right| d x d t .
\end{aligned}
$$

But

$$
\begin{aligned}
\iint_{D} \eta^{2} K \bar{u}^{-1}\left|\bar{u}_{t}\right| d x d t & =\left(\operatorname{sgn} \bar{u}_{t}\right) \iint_{D} \eta^{2} K(\log \bar{u})_{t} d x d t \\
& =-\left(\operatorname{sgn} \bar{u}_{t}\right) \iint_{D} 2 \eta \eta_{t} K \log \bar{u} d x d t \\
& \leq \iint_{D} 2 \eta\left|\eta_{t}\right| K|\log \bar{u}| d x d t
\end{aligned}
$$

since $\eta \in C_{0}^{1}(D)$, and hence

$$
\begin{aligned}
-\left.\int_{\Omega} \eta^{2} \log \bar{u}\right|_{t=\tau_{1}} ^{t=\tau_{2}} d x+\frac{1}{2} \iint_{D} \eta^{2} \bar{u}^{-2}\left|\bar{u}_{x}\right|^{2} d x d t & \\
& \leq \iint_{D} J d x d t+(2+2 K) \iint_{D} \eta\left|\eta_{t}\right| \log \bar{u} d x d t .
\end{aligned}
$$

Suppose that $\beta \neq-1$. Then if $v=\bar{u}^{r}, r=(\beta+1) / 2$, (3.18) becomes

$$
\begin{aligned}
(\operatorname{sgn} \beta)\left\{\left.(\beta+1)^{-1} \int \eta^{2} v^{2}\right|_{t=\tau_{1}} ^{t=\tau_{2}} d x+\gamma \beta\left(2 r^{2}\right)^{-1} \iint_{\eta^{2}}\left|v_{x}\right|^{2} d x d t\right\} \\
\leq\left\|\left[J+(2+2 K)|\beta+1|^{-1} \eta\left|\eta_{t}\right|\right] v^{2}\right\|_{1,1},
\end{aligned}
$$

where the $(x, t)$ integration is over $R(1) \times\left(\tau_{1}, \tau_{2}\right)$, and $J=F \eta^{2}+E\left|\eta_{x}\right|^{2}, F=$ $K^{2}|\beta|^{-1}+K\left(1-k^{-1}\right)$, and $E=4|\beta|^{-1}$.

The remainder of the proof is identical to that of Aronson and Serrin. The only difference between our fundamental inequalities (3.18), (3.19) and the corresponding ones of Aronson and Serrin is that inequalities (3.18) and (3.19) have the constant $2+2 \mathrm{~K}$ as a coefficient of the second term on the right-hand side instead of the constant 2. (See [3, pp. 103-110].)

4. The $\bar{C}_{2+a}$ spaces. We will assume the notation and description of $D$ given in $\$ 1$. We introduce the metric $d$ defined by

$$
d(P, Q)=\left[|x-\bar{x}|^{2}+|t-\bar{t}|\right]^{1 / 2},
$$

where $P=(x, t), Q=(\bar{x}, \bar{t})$, and $|x|=\left(\sum_{i=1}^{n} x_{i}^{2}\right)^{1 / 2}$. Hölder continuity of a function $f$ will be defined with respect to the metric $d$.

Suppose $0<a<1$, and let

$$
|u|_{0}^{D}=\sup _{D}|u|
$$




$$
\begin{aligned}
\bar{H}_{a}^{D}(u) & =\sup _{P, Q \in D} \frac{|u(P)-u(Q)|}{d(P, Q)^{\alpha}}, \\
|\bar{u}|_{a}^{D} & =|u|_{0}^{D}+\bar{H}_{a}^{D}(u), \\
\bar{C}_{a}(D) & =\left\{u: u: \bar{D} \rightarrow R,|\bar{u}|_{a}^{D}<\infty\right\} .
\end{aligned}
$$

Theorem 4.1. $\bar{C}_{a}(D)$ is a Banach space with norm $|-|_{a}^{D}$.

Proof. (See [6, pp. 62-63].)

Denote by $D_{x}^{m}$ any partial derivative of order $m$ with respect to the variables $x_{1}, \cdots, x_{n}$ and let $D_{t}=\partial / \partial t$. If $D_{x} u, D_{x}^{2} u, D_{t} u$ exist in $D$, then we define

$$
|\bar{u}|_{2+a}^{D}=|\bar{u}|_{a}^{D}+\sum\left|\overline{D_{x} u}\right|_{a}^{D}+\sum\left|\overline{D_{x}^{2} u}\right|_{a}^{D}+\left|\overline{D_{t} u}\right|_{a}^{D},
$$

where the sums are taken over all partial derivatives of the indicated order. Let

$$
\bar{C}_{2+a}(D)=\left\{u: u: \bar{D} \rightarrow R,|\bar{u}|_{2+a}^{D}<\infty\right\} .
$$

Theorem 4.2. $\bar{C}_{2+a}(D)$ is a Banach space with norm $|-|_{2+a}^{D}$.

Proof. (See [6].)

Definition 4.3. We say that $D$ has property $(\overline{\mathrm{E}})$ if for every point $Q$ of $\bar{S}$, there exists an $(n+1)$-dimensional neighborhood $V$ such that $V \cap \bar{S}$ can be represented, for some $i(1 \leq i \leq n)$, in the form $x_{i}=b\left(x_{1}, \cdots, x_{i-1}, x_{i+1}, \cdots, x_{n}, t\right)$, where $b, D_{x} b, D_{x}^{2} b, D_{t} b$ are Hölder continuous of exponent $a$.

Definition 4.4. If $D$ has property $(\overline{\mathrm{E}})$ and if the functions $D_{x} D_{t} b, D_{t}^{2} b$ of the local representations of $\bar{S}$ exist and are continuous functions, then we say that $D$ has the property $(\overline{\overline{\mathrm{E}}})$.

Definition 4.5. A function $\psi$ defined on $\bar{B}+S$ is said to belong to $\bar{C}_{2+a}(D)$ if there exist functions $\Psi$ in $\bar{C}_{2+a}(D)$ such that $\Psi=\psi$ on $\bar{B}+S$. Then $|\bar{\psi}|_{2+a}^{D}$ is defined by

$$
|\bar{\psi}|_{2+a}^{D}=\inf _{\Psi}|\bar{\Psi}|_{2+a}^{D}
$$

where the infimum is taken over all $\Psi \in \bar{C}_{2+a}(D)$ which coincide with $\psi$ on $\bar{B}+S$.

5. Refinement of the Friedman boundary estimates. For the case $c \equiv 1$, A. Friedman [6] obtains a priori boundary estimates for $u$ in terms of the $|\bar{u}|_{2+a}$ norms. We will state the theorem which he proves. Assume that the coefficients of $L$, defined in (1.1), are uniformly Hölder continuous (exponent $\alpha$ ) in $D$, that there exists a constant $\bar{K}_{1}$ such that $\left|\bar{a}^{i j}\right|_{a}^{D} \leq \bar{K}_{1},\left|\overline{b^{i}}\right|_{a}^{D} \leq \bar{K}_{1},|\bar{d}|_{a}^{D} \leq \bar{K}_{1}$, and $c \equiv 1$. Assume also that $|\bar{f}|_{\alpha}^{D}<\infty$ and that $u=\psi$ on $\bar{B}+S$. Furthermore, for any $(x, t) \in D$ and any real vector $\xi$, 


$$
\sum_{i=1}^{n} a^{i j}(x, t) \xi_{i} \xi_{j} \geq K_{2}|\xi|^{2}, \quad K_{2}>0 .
$$

We will refer to all the above conditions as conditions $(*)$.

Theorem 5.1 (Friedman). Let conditions (*) bold and assume that $D$ bas the property $(\overline{\mathrm{E}})$ and that $\psi \in \bar{C}_{2+\alpha}(D)$. There exists a constant $\bar{K}$ depending only on $\bar{K}_{1}, K_{2}, \alpha$, and $D$ such that if $u$ is a solution of the first initial-boundary value problem, $L u=f$ on $D+B_{T}, u=\psi$ on $\bar{B}+S$, and if $u \in \bar{C}_{2+a}(D)$, then

$$
|\bar{u}|_{2+a}^{D} \leq \bar{K}\left(|\overline{\bar{\psi}}|_{2+a}+|\bar{f}|_{a}\right) \text {. }
$$

We remark that $\bar{K}$ also depends on $c(x, t)$ being identically one.

Friedman then proves the following theorem.

Theorem 5.2. Let conditions (*) bold and assume that $D$ bas property $(\overline{\bar{E}})$, that $\psi \in \bar{C}_{2+a}(D)$, and that $L \psi=f$ on $\partial B$. Then there exists a unique solution $u$ of the first initial-boundary value problem, $L u=f$ on $D+B_{T}^{*} u=\psi$ on $\bar{B}+S$, and, furthermore, $u \in \bar{C}_{2+a}(D)$.

We shall consider the case $c(x, t) \geq \mu>0$ on $\bar{D}, \mu>1$, which is clearly equivalent to the case $c(x, t) \equiv 1$, and determine exactly how the constant $\mu$ appears in the estimate corresponding to (5.1). The estimates of Friedman are obtained via the study of a fundamental solution of the adjoint of the equation

$$
L u=\sum_{i=1}^{n} a^{i j}(x, t) u_{x_{i} x_{j}}-c(x, t) u_{t}=0 .
$$

Definition 5.3. A fundamental solution of $L u=0$ (in $\bar{D}$ ), $L$ defined in (1.1), is a function $\Gamma(x, t ; \xi, \tau)$ defined for all $(x, t) \in \bar{D},(\xi, \tau) \in \bar{D}, t>\tau$, which satisfies the following conditions:

$$
\begin{aligned}
& \text { for fixed }(\xi, \tau) \in \bar{D}, L(\Gamma(x, t ; \xi, \tau))=0, \\
& \text { for each }(x, t) \text { such that } x \in \Omega, t \in(\tau, T]
\end{aligned}
$$

$d \xi=d \xi_{1} \ldots d \xi_{n}$ and $\Omega$ is Lebesgue measurable.

We will state the theorems analogous to the case $c(x, t) \equiv 1$ which are proved in [6]. The proofs are straightforward and generally the same as the case $c(x, t) \equiv 1$.

The procedure which Friedman uses to establish a fundamental solution is the parametrix method due to E. E. Levi[11]. Assume as previously that $L$ is the operator (1.1) where

$$
\lambda_{0}|\xi|^{2} \leq \sum_{i, j=1}^{n} a^{i j}(x, t) \xi_{i} \xi_{j} \leq \lambda_{1}|\xi|^{2}
$$


for some positive constants $\lambda_{0}$ and $\lambda_{1}$, where $(x, t) \in \bar{D}$ and $\xi=\left(\xi_{1}, \cdots, \xi_{n}\right)$ is a nonzero real vector.

Assume that the coefficients of $L$ are continuous and satisfy, for all $(x, t)$, $\left(x^{0}, t^{9}\right) \in \bar{D}$,

$$
\left\{\begin{array}{l}
\left|a^{i j}(x, t)-a^{i j}\left(x^{0}, t^{0}\right)\right| \leq C\left(\left|x-x^{0}\right|^{a}+\left|t-t^{0}\right|^{a / 2}\right), \\
\left|b^{i}(x, t)-b^{i}\left(x^{0}, t^{0}\right)\right| \leq C\left|x-x^{0}\right|^{a} \\
\left|d(x, t)-d\left(x^{0}, t^{0}\right)\right| \leq C\left|x-x^{0}\right|^{\alpha} \\
\left|c(x, t)-c\left(x^{0}, t^{0}\right)\right| \leq C\left(\left|x-x^{0}\right|^{a}+\left|t-t^{0}\right|^{\alpha / 2}\right),
\end{array}\right.
$$

$c(x, t) \geq \mu>0$, where $\mu>1$.

Denote the inverse of the matrix $\left(a^{i j}(x, t)\right)$ by $\left(\bar{a}^{i j}(x, t)\right)$; and let

$$
Q^{y, \sigma}(x, \xi)=\sum_{i, j=1}^{n} \bar{a}^{i j}(y, \sigma)\left(x_{i}-\xi_{i}\right)\left(x_{j}-\xi_{j}\right),
$$

where $y=\left(y_{1}, \cdots, y_{n}\right)$. Then

$$
\bar{\lambda}_{0}|x-\xi|^{2} \leq Q^{y, \sigma}(x, \xi) \leq \bar{\lambda}_{1}|x-\xi|^{2},
$$

and

$$
\left|\bar{a}^{i j}(x, t)-\bar{a}^{i j}\left(x^{0}, t^{0}\right)\right| \leq \bar{C}\left(\left|x-x^{0}\right|^{a}+\left|t-t^{0}\right|^{a / 2}\right)
$$

where $\bar{\lambda}_{0}, \bar{\lambda}_{1}, \bar{C}$ are positive constants depending only on $\lambda_{0}, \lambda_{1}$, and $C$.

Now suppose that $t>\tau$ and define

$$
w^{y, \sigma}(x, t ; \xi, \tau)=(t-\tau)^{-n / 2} \exp \left\{-\frac{c(y, \sigma) Q^{y, \sigma}(x, \xi)}{4(t-\tau)}\right\},
$$

and

$$
Z(x, t ; \xi, \tau)=C(\xi, \tau) w^{\xi, \tau}(x, t ; \xi, \tau)
$$

where

$$
C(x, t)=\left[c(x, t)(4 \pi)^{-n} \operatorname{det}\left(\bar{a}^{i j}(x, t)\right)\right]^{1 / 2} .
$$

It is then routine to show that for each fixed $(\xi, \tau), Z(x, t ; \xi, \tau)$ satisfies the equation $L_{0} u=0$ as a function of $(x, t)$ for $L_{0}$ given by

$$
L_{0} u=\sum_{i, j=1}^{n} a^{i j}(\xi, \tau) u_{x_{i} x_{j}}-c(\xi, \tau) u_{t} \text {. }
$$

Theorem 5.4. Let $f \in C(\bar{D})$. Then

$$
J(x, t, \tau)=\int_{\Omega} Z(x, t ; \xi, \tau) f(\xi, \tau) d \xi
$$

is a continuous function in $(x, t, \tau), x \in \bar{\Omega}, 0 \leq \tau<t \leq T$, and

$$
\lim _{\tau \rightarrow t} J(x, t, \tau)=f(x, t)
$$


uniformly with respect to $(x, t), x \in B, 0<t \leq T$, where $B$ is any closed subset of $\Omega$.

Proof. The proof is a slight modification of $[6$, pp. 4-6].

Then $Z$ satisfies (5.3) and is therefore a fundamental solution of $L_{0} u=0$. $L_{0}$ is regarded as a first approximation to $L$, and regarding $Z$ as a principal part of the fundamental solution $\Gamma$ to $L u=0$, we seek to determine $\Gamma$ in the form

(5.13) $\quad \Gamma(x, t ; \xi, \tau)=Z(x, t ; \xi, \tau)+\int_{\tau}^{t} \int_{\Omega} Z(x, t ; \eta, \sigma) \Phi(\eta, \sigma ; \xi, \tau) d \eta d \sigma$.

$\Phi$ will be determined by the requirement that $L \Gamma=0$ 。

By using volume potentials, it can be shown that (see [6]) solving the equation $L \Gamma=0$ is equivalent to solving the Volterra integral equation

(5.14) $\Phi(x, t ; \xi, \tau)=L Z(x, t ; \xi, \tau)+\int_{\tau}^{t} \int_{\Omega} L Z(x, t ; y, \sigma) \Phi(y, \sigma ; \xi, \tau) d y d \sigma$.

One shows that there exists a solution $\Phi(x, t ; \xi, \tau)$ to $(5.14)$ given by

where

$$
\Phi(x, t ; \xi, \tau)=\sum_{\nu=1}^{\infty}(L Z)_{\nu}(x, t ; \xi, \tau)
$$

$$
(L Z)_{1}=L Z
$$

and

$$
L Z_{\nu+1}(x, t ; \xi, \tau)=\int_{\tau}^{t} \int_{\Omega}[L Z(x, t ; y, \sigma)](L Z)_{\nu}(y, \sigma ; \xi, \tau) d y d \sigma .
$$

An argument similar to that of Friedman shows that the series converges and that

$$
|\Phi(x, t ; \xi, \tau)| \leq \mu^{-1 / 2} C(t-\tau)^{-\beta}|x-\xi|^{-(n+2-2 \beta-a)} \exp \left[-\frac{\mu^{1 / 2} \lambda_{0}^{*}|x-\xi|^{2}}{4(t-\tau)}\right]
$$

$$
\leq \mu^{-1 / 2} C(t-\tau)^{-\beta}|x-\xi|^{-n-2+2 \beta+\alpha},
$$

for $0 \leq \beta \leq(n+2-\alpha) / 2$, where $\lambda_{0}^{*}$ is any real number less than $\lambda_{0}, t-\tau>0$, and $C$ is a constant independent of $\mu$.

Theorem 5.5. $\Phi(x, t ; \xi, \tau)$ is Hölder continuous in $x$. For any $0<\beta<\alpha$,

$$
\begin{aligned}
& |\Phi(x, t ; \xi, \tau)-\Phi(y, \sigma ; \xi, \tau)| \\
& \leq \mu^{-1 / 2} C|x-y|^{\beta}(t-\tau)^{-(n+2-\gamma) / 2} \\
& \quad\left\{\exp \left[-\frac{\mu^{1 / 2} \lambda^{*}|x-\xi|^{2}}{t-\tau}\right]+\exp \left[-\frac{\mu^{1 / 2} \lambda^{*}|y-\xi|^{2}}{t-\tau}\right]\right\},
\end{aligned}
$$


where $\gamma=\alpha-\beta, \lambda^{*}$ is a positive constant, and $t-\tau>0$.

Proof. (See [6, p. 17].) One must, of course, keep track of $\mu^{1 / 2}$.

For the equation $L u=0$, where $L$ is as defined in (1.1), one can write the adjoint equation

$$
L^{*} v=\sum_{i, j=1}^{n} a^{i j}(x, t) v_{x_{i} x_{j}}+\sum_{i=1}^{n} b^{i *} v_{x_{i}}+c^{*} v_{t}+d^{*} v=0
$$

where $b^{i^{*}}=-b^{i}+2 \sum_{j=1}^{n} a_{x_{j}}^{i j}, c^{*}=c-2 \sum_{j=1}^{n} a_{x_{j}}^{i j} d^{*}=d-\sum_{i=1}^{n} b_{x_{i}}^{i}+\sum_{i, j=1}^{n} a_{x_{i} x_{j}}^{i j}$ $+c_{t}$.

For $u, v \in C^{2,1}(\bar{D})$, Green's identity,

$$
v L u-u L^{*} v=\sum_{i=1}^{n}\left[\sum_{j=1}^{n}\left(v a^{i j} u_{x_{j}}-u a^{i j} v_{x_{j}}-u v a_{x_{j}}^{i j}\right)+b^{i} u v\right] x_{j}-(c u v)_{t},
$$

can be easily verified.

If $u$ and $v$ have compact support in $D$, the integration of both sides of (5.18) yields

$$
\iint_{\bar{D}}\left(v L u-u L^{*} v\right) d x d t=0 .
$$

Definition 5.6. A fundamental solution of $L^{*} v=0$ is a function $\Gamma^{*}(x, t ; \xi, \tau)$ defined for all $(x, t),(\xi, \tau) \in \bar{D}, t<\tau$, satisfying

$$
\begin{aligned}
& \text { if }(\xi, \tau) \text { is fixed, } L^{*} \Gamma^{*}=0 \text { as a function of }(x, t), \\
& x \in \Omega, t \in[0, \tau) \text {; }
\end{aligned}
$$

and

$$
\begin{aligned}
& \text { if } f \in C(\bar{D}), \text { for each } x \in \bar{\Omega}, \\
& \lim _{t \rightarrow \tau^{-}} \int_{\Omega} \Gamma^{*}(x, t ; \xi, \tau) f(\xi) d \xi=f(x) .
\end{aligned}
$$

Using the same method as for $L u=0$, we can construct a fundamental solution $\Gamma^{*}$ of $L^{*} v=0$. The parametrix $Z^{*}(x, t ; \xi, \tau)$ is analogous to $Z(x, t ; \xi, \tau)$, namely,

$$
Z^{*}(x, t ; \xi, \tau)=C(x, t)(\tau-t)^{-n / 2} \exp \left\{-\frac{c(x, t) Q^{x, t}(x, \xi)}{4(\tau-t)}\right\}, \quad t<\tau
$$

$Z^{*}$ satisfies $L_{0}^{*} v=0$, where $L_{0}$ is defined in (5.12).

The estimates

$$
\left|\Gamma^{*}(x, t ; \xi, \tau)\right| \leq \mu^{-1 / 2} C(\tau-t)^{-n / 2} \exp \left\{-\frac{\mu \lambda_{0}^{*}|x-\xi|^{2}}{4(\tau-t)}\right\}
$$

and 
(5.24) $\left|D_{\tau}^{k} D_{\xi}^{j} \Gamma^{*}(x, t ; \xi, \tau)\right| \leq \mu^{-1 / 2} C(\tau-t)^{-(n+2 k+j) / 2} \exp \left\{-\frac{K \mu|x-\xi|^{2}}{\tau-t}\right\}, \mu \lessdot 1$, can be verified in the usual manner.

Suppose that $N$ is a fixed semicube with top $P=\left(x^{0}, t^{9}\right)$ and edge $d$; that is, $N$ is defined by the inequalities

$$
\begin{aligned}
& x_{i}^{0}-d \leq x_{i} \leq x_{i}^{0}+d, \quad i=1, \cdots, n, \\
& t^{0}-d^{2} \leq t \leq t^{0} .
\end{aligned}
$$

Note that if $Q \in N, d(P, Q) \leq(n+1)^{1 / 2} d$.

Let $H_{Q, N}[g]$ be defined by

$$
H_{Q, N}[g]=\sup _{R \in N} \frac{|g(Q)-g(R)|}{d(Q, R)^{\alpha}} .
$$

Using the preceding estimates for $\Gamma^{*}(x, t ; \xi, \tau)$, we can prove the following the orem.

Theorem 5.7. Let $f$ and $c, c \geq \mu>0$, be Hölder continuous (exponent $\alpha$ ) in N. Suppose that $a^{i j}$ is constant for each $i, j$, that $\left|a^{i j}\right| \leq k_{1}$, and that $\sum_{i, j=1}^{n} a^{i j} \xi_{i} \xi_{j} \geq k_{2}|\xi|^{2}, k_{2}>0$. Furthermore, let $u, u_{x_{i}} u_{x_{i} x_{j}}$ be Hölder continuous (of exponent $\alpha$ ) in N. If $u$ satisfies

$$
L u=\sum_{i, j=1}^{n} a^{i j} u_{x_{i} x_{j}}-c u_{t}=f
$$

in $N$, then there is a constant $K$ depending only on $n, a, k_{1}, k_{2}$ such that for $i=0,1,2$,

(5.27) $\left|D_{x}^{i} u(P)\right| \leq \mu^{-1 / 2} K\left[d^{-i} \sup _{N}|u|+d^{2-i} \sup _{N}|f|+d^{2-i+a} H_{P, N}[f]\right] \equiv \mu^{-1 / 2} K I_{i}$ and

$$
\frac{d^{\alpha}\left|D_{x}^{i} u(P)-D_{x}^{i} u(Q)\right|}{d(P, Q)^{\alpha}} \leq \mu^{-1 / 2} K\left[I_{i}+d^{2-i+a} H_{Q, N}[f]\right]
$$

if $d(P, Q) \leq d / 4$.

Proof. The proof is given in [6]. We need be concerned with the constant only.

The main theorem which we shall need is the following.

Theorem 5.8. Suppose that the coefficients of $L$, defined in (1.1), are uni-

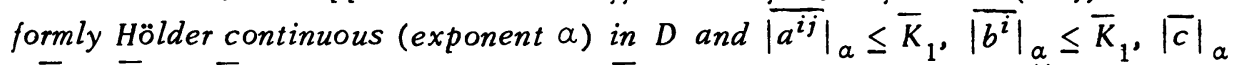
$\leq \bar{K}_{1},|\bar{d}|_{a} \leq \bar{K}_{1}$, that $c \geq \mu>0$, that $|\bar{f}|_{a}<\infty$, and that $\sum_{i, j=1}^{n} a^{i j} \xi_{i} \xi_{j} \geq K_{2}|\xi|^{2}$, $K_{2}>0$. Assume that $D$ bas property $(\bar{E})$ and that $\psi \in \bar{C}_{2+a}(D)$.

Then there exists a constant $\bar{K}$ depending only on $\bar{K}_{1}, K_{2}, a$, and $D$ such 
that if $u$ is a solution to $L u=f$, with $u=\psi$ on $\bar{B}+S$, and if $u \in \bar{C}_{2+a}(D)$, then

$$
|\bar{u}|_{2+a} \leq \mu^{-1 / 2} \bar{K}\left(|\bar{\psi}|_{2+a}+|\bar{f}|_{a}\right) \text {. }
$$

Proof. The proof follows directly from the proofs in [6], and the more refined estimates (5.24).

6. Uniform estimates for $\left|\overline{u_{t}}\right|_{a}$. First, we will show that $u_{t}$ is uniformly bounded, independent of the minimum of $c$ on $D$. Then, knowing that $u_{t}$ is uniformly bounded, we can estimate the Hölder coefficient of $u_{t}$, using the Harnack inequality derived in $\$ 3$.

Theorem 6.1. Assume that $a^{i j}$ is constant for each $i, j$, that all coefficients of $L$, defined in (1.1), are of class $C^{1,1}(\bar{D}), u \in C(\bar{D}), u \in C^{3}(D)$, and $a^{11} \lambda^{2}$ $+b^{1} \lambda \geq 1$ for some $\lambda>0$. Suppose, further, that the coefficients of $L$ are uniformly Hölder continuous (exponent $a$ ) in $D,\left|\overline{a^{i j}}\right|_{a} \leq \bar{K}_{1},\left|\overline{b^{i}}\right|_{a} \leq \bar{K}_{1},|\bar{c}|_{a} \leq \bar{K}_{1}$, $|\bar{d}|_{a} \leq \bar{K}_{1}$, that $m(c ; \bar{B}+S) \geq \mu>0$, that $|\bar{f}|_{a_{-}}<\infty$, and that $\sum_{i, j=1}^{n} a^{i j} \xi_{i} \xi_{j} \geq$ $K_{2}|\xi|^{2}, K_{2}>0$. Assume that $D$ has property $(\overline{\mathrm{E}})$, and that $\psi \in \bar{C}_{2+a}(D)$.

Then there exist positive constants $C$ and $\bar{K}$, where $C$ depends only on $\lambda$, $M(|\beta| ; \bar{D}), \beta \in\left\{b_{t}, c_{t}, d_{x_{i}}, b_{x_{i}}, c_{x_{i}}\right\}$ and $\bar{K}$ depends only on $\bar{K}_{1}, K_{2}, \alpha$, and $D$, such that if $u$ is a solution to $L u=f$, with $u=\psi$ on $\bar{B}+S$, and if $u \in \bar{C}_{2+a}(D)$, then

$M\left(\left|u_{t}\right| ; \bar{D}\right) \leq C\left[\hat{\mu}^{-3 / 2} K\left(|\bar{\psi}|_{2+a}+|\bar{f}|_{\alpha}\right)+M\left(\left|f_{t}\right| ; \bar{D}\right)+\sum_{i=1}^{n} M\left(\left|f_{x_{i}}\right| ; \bar{D}\right)+M(|f| ; \bar{D})\right]$, for some $\hat{\mu}$ which is independent of $m(c ; D)$.

Proof. Since $m(c ; \bar{B}+S) \geq \mu>0$, there exists an $\epsilon$-strip, $(\bar{B}+S)$, about $\bar{B}+S$ such that $(\bar{B}+S)_{\epsilon} \subset \bar{D}$ and $m\left(\bar{c}(\overline{\bar{B}}+S)_{\epsilon}\right)>0$. Thus, $m\left(c ; \overline{\bar{B}+S)_{\epsilon}}\right) \geq$

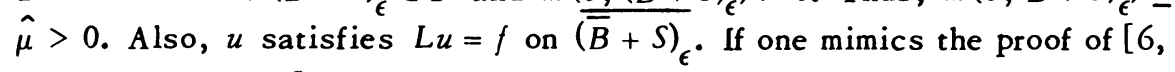
Theorem 4, p. 121], it can be shown that

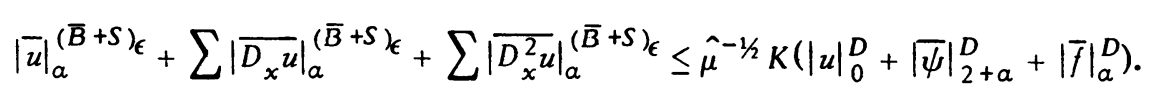

Since $u$ satisfies $L u=f$ and $c$ is bounded away from zero, one can solve the differential equation over $(\bar{B}+S)_{\epsilon}$ for $D_{t} u$ in terms of $u, D_{x} u$ and $D_{x}^{2} u$ and obtain a similar bound on $D_{t} u$. Solving the differential equation for $D_{t} u$ gives $\hat{\mu}^{-3 / 2}$ in the estimate. Corollary 2.7 guarante es that $|u|_{0}^{D} \leq C\left(M(|\psi| ; D)+|f|_{0}\right)$ for some constant $C$. Hence we obtain an estimate for $\left|u_{t}\right|_{a}^{(\bar{B}+S)} \epsilon$.

It is clear that

$$
\begin{aligned}
M(|u| ; & \bar{B}+S)+M\left(\left|u_{t}\right| ; \bar{B}+S\right)+\sum_{i=1}^{n} M\left(\left|u_{x_{i}}\right| ; \bar{B}+S\right) \\
& \leq M\left(|u| ;(\bar{B}+S)_{\epsilon}\right)+M\left(\left|u_{t}\right| ;(\bar{B}+S)_{\epsilon}\right)+\sum_{i=1}^{n} M\left(\left|u_{x_{i}}\right| ;(\bar{B}+S)_{\epsilon}\right) .
\end{aligned}
$$


We have already proved that the right-hand side of the above

$$
\leq \hat{\mu}^{-3 / 2} K\left(|u|_{0}^{D}+|\bar{\psi}|_{2+a}^{D}+|\bar{f}|_{a}^{D}\right)
$$

which is

$$
\leq \hat{\mu}^{-3 / 2} \bar{K}\left(|\bar{\psi}|_{2+a}+|\bar{f}|_{a}\right) .
$$

Applying Theorem 2.8, the desired inequality follows.

We have now shown that for constant $a^{i j}$ and $c>0$ on $\bar{B}+S,\left|u_{t}\right|$ is uniformly bounded on $\bar{D}$. Since $\left|u_{t}\right|$ is bounded, independent of $m(c ; D)$, we can proceed with estimating the Hölder coefficient (exponent $a$ ) of $u_{t}$. Recall that if $a^{i j}$ is constant for each $i, j$, and $u$ satisfies $L u=f$, where $L$ is defined as in (1.1), then $u_{t}$ satisfies

$$
\left(L-c_{t}\right) u_{t}=f_{t}-\sum_{i=1}^{n} b_{t}^{i} u_{x_{i}}-d_{t} u .
$$

Theorem 6.2. Suppose that $u_{t}$ is a bounded nonnegative classical solution of (6.1) in D, where $a^{i j}$ is constant for each $i, j$. Suppose that all coefficients of $L$ are of class $C^{1,1}(\bar{D}), u \in C(\bar{D}), u \in C^{3}(D)$, and $a^{1{ }^{1}} \lambda^{2}+b^{1} \lambda \geq 1$ for some $\lambda>0$. Suppose that the functions $u, u_{t}, a^{i j}, b^{i}, c, d, f_{t}, \sum_{i=1}^{n} b_{x_{i}}^{i} c_{t^{\prime}}$ and $d_{t}$ satisfy the property that their absolute values are uniformly bounded by some constant. If $D(3 \rho) \subset D$, then

$$
\underset{D^{*}(\rho)}{\operatorname{ess} \max } u_{t} \leq C\left(\begin{array}{l}
\text { ess min } \\
D(\rho)
\end{array}\left(u+\rho^{\theta} k\right)\right),
$$

where $C$ is a positive constant depending only on $\rho$ and the structure of (6.1), $\theta$ is defined in (3.8), and $k=\left\|f_{t}\right\|+\left\|d_{t} u\right\|+\|1-c\|$. Furthermore, $C$ is independent of $\left\|f_{t}\right\|,\left\|d_{t}\right\|,\|1-c\|$.

Proof. The equation (6.1) can be rewritten in the form

$$
\begin{aligned}
& \sum_{i, j=1}^{n}\left\{a^{i j}\left(u_{t}\right)_{x_{i}}\right\}_{x_{j}}+\sum_{i=1}^{n} b^{i}\left(u_{t}\right)_{x_{i}}+(1-c)\left(u_{t}\right)_{t} \\
& \quad+\left(d-c_{t}\right) u_{t}-\sum_{i=1}^{n} b_{t}^{i} u_{x_{i}}-\left(d_{t} u+f_{t}\right)=\left(u_{t}\right) .
\end{aligned}
$$

The structure inequalities

$$
\begin{aligned}
p \cdot A(x, t, p) & \geq \gamma|p|^{2}, \\
\left|B\left(x, t, u_{t}, p, q\right)\right| & \leq C\left(|p|+|q|+\left|u_{t}\right|+1\right), \\
|A(x, t, p)| & \leq C|p|
\end{aligned}
$$

are obviously satisfied, with $A(x, t, p)$ defined as before in (3.1) and 
$B\left(x, t, u_{t},\left(u_{t}\right)_{x},\left(u_{t}\right)_{t}\right)=b\left(u_{t}\right)_{x}+(1-c)\left(u_{t}\right)_{t}+\left(d-c_{t}\right) u_{t}+b_{t} u_{x}-\left(d_{t} u+f_{t}\right)$. Now apply Theorem 3.1 .

Theorem 6.3. Suppose that $u$ satisfies the bypotheses of Theorems 6.1 and 6.2. Suppose that $Q=(x, t), P=(y, s)$ are points in $D$ with $s \leq t$. Then

$$
\left|u_{t}(P)-u_{t}(Q)\right| \leq H\left(\max _{\bar{B}+S}|\psi|+k\right)(d(P, Q) / R)
$$

where $H$ and $\alpha(0<a<1)$ are positive constants depending only on the structure of (6.1), $k=\left\|f_{t}\right\|+\left\|d_{t} u\right\|+\|1-c\|$, and $R=\min \{d(Q, \bar{B}+S), 1\}$.

Proof. Since $u$ is a solution to the first initial-boundary value problem, a corollary to the weak maximum principle, Corollary 2.7 , implies that $|u|$ is uniformly bounded on $\bar{D}$, independent of the minimum of $c$ on $D$. Theorem 6.1 assures that $\left|u_{t}\right|$ is uniformly bounded on $\bar{D}$, independent of $m(c ; D)$. Corollary 2.9 together with the argument of Theorem 6.1 implies that $\left|u_{x}\right|$ is uniformly bounded on $\bar{D}$, likewise independent of $m(c ; D)$. This theorem is analogous to Theorem 4 of $[3, \mathrm{p} .110]$ of Aronșon and Serrin, which holds for $c(x, t) \equiv 1$. We use Theorem 6.2 in place of their analogue, Theorem 3, and the proof is identical.

We have thus established interior Hölder continuity for $u_{t}$ and have shown that on a compact set in the interior of $D$, the Hölder coefficient is uniformly bounded independent of $m(c ; D)$. We now establish uniform Hölder continuity of $u_{t}$ in $\bar{D}$.

Theorem 6.4. Assume that the conditions of Theorem 6.3 bold. Then $u_{t}$ is uniformly Hölder continuous (exponent $\alpha$ ) in $\bar{D}$ and the Hölder coefficient of $u_{t}$ is bounded independent of $m(c ; D)$.

Proof. The proof is similar to that of Theorem 6.1. Since $m(c ; \bar{B}+S) \geq$ $\mu>0$, there exists an $\epsilon$-strip (measured in the metric $d),(\bar{B}+S) \epsilon$, about $\bar{B}+S$, $(\bar{B}+S)_{\epsilon} \subset \bar{D}$, such that $m\left(c ;(\bar{B}+S)_{\epsilon}\right) \geq \mu>0$. Again, the proof by Friedman $\left[6\right.$, Theorem 4, p. 121] holds since $c$ is bounded away from zero on $(\bar{B}+S) \epsilon^{\cdot}$. As in the proof of Theorem 6.1 , we see that

$$
|u|_{a}^{(\bar{B}+S) \epsilon}+\left|\overline{D_{x} u}\right|_{a}^{(\bar{B}+S) \epsilon}+\left|\overline{D_{x}^{2} u}\right|_{a}^{(\bar{B}+S) \epsilon} \leq \hat{\mu}^{-3 / 2} K\left(|u|_{0}^{D}+|\bar{\psi}|_{2+a}+|\bar{f}|_{a}\right) .
$$

Solve $L u=f$ for $u_{t}$ in $(\bar{B}+S)_{\epsilon}$. Then

$$
\left|\bar{u}_{t}\right|_{a}^{(\bar{B}+S)_{\epsilon}} \leq \hat{\mu}^{-3 / 2} K\left(M(|\psi| ; D)+|f|_{0}+|\bar{\psi}|_{2+a}+|\bar{f}|_{a}\right) .
$$

Therefore,

$$
\bar{H}_{a}^{(\bar{B}+S)} \epsilon\left(u_{t}\right) \leq \hat{\mu}^{-3 / 2} \bar{K}\left(|\bar{\psi}|_{2+a}+|\bar{f}|_{a}\right)
$$


Let $D_{\epsilon}$ be the complement of $(\bar{B}+S)_{\epsilon}$ in $\bar{D}$. Theorem 6.3 implies $\bar{H}_{a}^{D} \epsilon\left(u_{t}\right)$ $\leq C / \epsilon^{a}$ for some constant $C$. Hence, $u_{t}$ is uniformly Hölder continuous (exponent $\alpha)$ in $\bar{D}$ and the Hölder coefficient is bounded independent of $m(c ; D)$.

We now proceed to show existence of a solution to the first initial-boundary value problem in case the conditions of Theorems 6.3-6.4 hold.

7. A classical (strong) solution. This section shall be devoted to proving the existence of a unique solution to the first initial-boundary value problem for the nonuniformly parabolic operator (1.1) where $a^{i j}$ is constant for each $i, j$. Assume that all the conditions mentioned in Theorems 6.3-6.4 are satisfied. Assume also that $D$ has property $(\overline{\bar{E}})$; see Definition 4.4 .

The method of proof involves perturbing the coefficient $c$. We shall use the sharper Schauder-type estimates given in $\oint_{5}$ as well as the estimate for $\left|\bar{u}_{t}\right|^{D}$, which is independent of $m(c ; D)$, to obtain a Cauchy sequence of solutions $\left\{u_{n}\right\}$ which converges to a classical solution. The following existence theorem for $c(x, t) \equiv 1$ is due to Friedman $[6$, p. 65].

Theorem 7.1. Assume that the conditions (*) of Theorem 5.1 are satisfied, that $D$ has property $(\overline{\overline{\mathrm{E}}})$, that $\psi \in \bar{C}_{2+\alpha}(D)$, and that $L \psi=f$ on $\partial B$. Then there exists a unique solution $u$ of the first initial-boundary value problem $L u=f$ on $D+B_{T}, u=\psi$ on $\bar{B}+S$ and, furthermore, $u \in \bar{C}_{2+a}(D)$.

Remark. Theorem 7.1 holds for $c(x, t) \geq \mu>0$, which is clearly equivalent to the case $c(x ; t) \equiv 1$.

We now solve the first initial-boundary value problem

$$
\begin{aligned}
L u & =\sum_{i, j=1}^{n} a^{i j} u_{x_{i}{ }_{j}}+\sum_{i=1}^{n} b^{i}(x, t) u_{x_{i}}-c(x, t) u_{t}+d(x, t) u \\
& =f \text { on } D+B_{T}, \quad u=\psi \quad \text { on } \bar{B}+S,
\end{aligned}
$$

without assuming that $c$ has a positive minimum in $D$.

Perturb the coefficient $c$ by $1 / k$ and consider the problem

$$
L^{k} u=L u-(1 / k) u_{t}=f \text { on } D+B_{T}, \quad u=\psi \quad \text { on } \bar{B}+S \text {. }
$$

By Theorem 7.1, there exists a unique solution $u_{k} \in \bar{C}_{2+a}(D)$ of (7.2).

We wish to show that the sequence $\left\{u_{k}\right\}$ obtained in this manner is Cauchy in the Banach space $\bar{C}_{2+a}(D)$. Consider, for $m>n, u_{n}-u_{m} \cdot u_{n}-u_{m}$ satisfies

$$
\begin{aligned}
& \sum_{i, j=1}^{n} a^{i j}\left(u_{n}-u_{m}\right)_{x_{i} x_{j}}+\sum_{i=1}^{n} b^{\dot{u}}(x, t)\left(u_{n}-u_{m}\right)_{x_{i}} \\
& +d(x, t)\left(u_{n}-u_{m}\right)-[c(x, t)+(1 / n)]\left(u_{n}-u_{m}\right)_{t} \\
& \text { with } u_{n}-u_{m}=0 \text { on } \bar{B}+S \text {. } \\
& =[(1 / n)-(1 / m)]\left(u_{m}\right)_{t} \text { on } D+B_{T} \text {, }
\end{aligned}
$$


Applying Theorem 5.8, we have the estimate

$$
\left|\overline{u_{n}-u_{m}}\right|_{2+a}^{D} \leq n^{1 / 2} \bar{K}|(1 / n)-(1 / m)|\left|\overline{\left(u_{m}\right)_{t}}\right|_{a} \leq n^{1 / 2}|(1 / n)-(1 / m)| \bar{K} M,
$$

for some constant $M$ which is independent of $n$ and $m$. The latter inequality follows from $\$ 6$. Since $m>n$,

$$
n^{1 / 2}|(1 / n)-(1 / m)| \leq n^{-1 / 2}+m^{-1 / 2}
$$

Hence, $\lim _{m, n \rightarrow \infty}\left|\overline{u_{n}-u_{m}}\right|_{2+\alpha}^{D}=0$. We have shown, therefore, that the sequence $\left\{u_{k}\right\}$ is Cauchy. Since $\bar{C}_{2+a}(D)$ is a Banach space, $\left\{u_{k}\right\} \rightarrow u$ in $|\cdot|_{2+a}^{D}$ for some $u \in \bar{C}_{2+a}(D)$. That is, $\left\{u_{n}\right\}$ converges to $u$ and the sequences of derivatives converge to the corresponding derivatives of $u$.

Since $u_{n}$ satisfies $L^{n} u_{n}=f$ in $D+B_{T}, u_{n}=\psi$ in $\bar{B}+S$, we let $n \rightarrow \infty$ and obtain that $u$ satisfies (7.1).

Uniqueness follows in the usual manner. Apply Corollary 2.7 to the difference $u-v$ of $u$ and $v$ where $u$ and $v$ both satisfy (7.1).

We have thus proved the main theorem.

Theorem 7.2. Assume that the conditions of Theorems 6.3 and 6.4 are satisfied, that $D$ bas property $(\overline{\bar{E}})$, that $\psi \in \bar{C}_{2+\alpha}(D)$, and that $L \psi=f$ on $\partial B$. Then there exists a unique solution $u$ of the first initial-boundary value problem (7.1) and, furthermore, $u \in \bar{C}_{2+a}(D)$.

Certain problems can be reduced to the case $a^{i j}$ constant. If one can show that $u_{t}$ is bounded, independent of the minimum of $c$ in $D$, then the techniques we have for estimating the Hölder coefficient of $u_{t}$ do not require the $a^{i j}$ to be constant.

Friedman derives estimates for the case $a^{i j}$ constant and then uses a perturbation technique to derive estimates for the general case. We cannot do the same because our estimates are not in such a usable form. It seems reasonable to expect that the case $a^{i j}$ constant can be used to obtain the more general result.

In the special case of one space variable, $L u=f$, where $L$ is defined by (1.1), becomes

$$
a(x, t) u_{x x}+b(x, t) u_{x}-c(x, t) u_{t}+d(x, t) u=f(x, t) .
$$

Since $a(x, t)$ is bounded away from zero, the above equation is equivalent to

$$
u_{x x}+\frac{b(x, t)}{a(x, t)} u_{x}-\frac{c(x, t)}{a(x, t)} u_{t}+\frac{d(x, t)}{a(x, t)} u=\frac{f(x, t)}{a(x, t)},
$$

and Theorem 7.2 guarantees existence of a unique solution to the first initialboundary value problem. 


\section{REF ERENCES}

1. D. G. Aronson, Non-negative solutions of linear parabolic equations, Ann. Scuola Norm. Sup. Pisa 29 (1968), 607-694.

2. - Regularity properties of flows through porous media, SIAM J. Appl. Math. 17 (1969), 461-467. MR 40 \#571.

3. D. G. Aronson and J. Serrin, Local behavior of solutions of quasilinear parabolic equations, Arch. Rational Mech. Anal. 25 (1967), 81-122. MR 39 \#5952.

4. W. T. Ford, Elements of simulation of fluid flow in porous media, Texas Tech University Press, Lubbock, Tex., 1971.

5. - The first initial boundary value problem for a nonuniform parabolic equation, J. Math. Anal. Appl. 3 (1972).

6. A. Friedman,Partial differential equations of parabolic type, Prentice-Hall, Englewood Cliffs, N. J., 1964. MR 31 \#6062.

7. C. D. Hill, A sharp maximum principle for degenerate elliptic-parabolic equations (to appear).

8. J. J. Kohn and L. Nirenberg, Degenerate elliptic-parabolic equations of second order, Comm. Pure Appl. Math. 20 (1967), 797-872. MR 38 \#2437.

9. O. A. Ladyženskaja, V. A. Solonnikov and N. N. Ural' ceva, Linear and quasilinear equations of parabolic type, "Nauka", Moscow, 1967; English transl., Transl. Math. Monographs, vol. 23, Amer. Math. Soc., Providence, R.I., 1968. MR 39 \#3159a, b.

10. O. A. Ladyženskaja and N. N. Ural' ceva, On Hölder continuity of solutions and their derivatives of linear and quasilinear elliptic and parabolic equations, Trudy Mat. Inst. Steklov. 73 (1964), 172-220; English transl., Amer. Math. Soc. Transl. (2) 61 (1967), 207-269. MR 30 \#4063.

11. E. E. Levi, Sulle equazioni lineari totalmente ellittiche alle derivate parziali, Rend. Circ. Mat. Palermo 24 (1907), 275-317.

12. J. Moser, A Harnack inequality for parabolic differential equations, Comm. Pure Appl. Math. 17 (1964), $101-134$; correction, ibid. 20 (1967), 231-236. MR 28 \#2357; MR 34 \#3121.

13. O. A. Olě̆nik, Quasi-linear second-order parabolic equations with many independent variables, Seminari 1962/63 Anal. Alg. Geom. e Topol., vol. 1, Ist. Naz. Alta Mat., Edizioni Cremonese, Rome, 1965, pp. 332-354. MR 33 \#2969.

14. O. A. Ole İnik and S. N. Kružkov, Quasi-linear parabolic second-order equations with several independent variables, Uspehi Mat. Nauk 16 (1961), no. 5 (101), 115-155= Russian Math. Surveys 16 (1961), no. 5, 105-146. MR 25 \#5289.

15. M. H. Protter and H. F. W. Wein berger, Maximum principles in differential equations, Prentice-Hall, Englew ood Cliffs, N. J., 1967. MR 36 \#2935.

16. N. S. Trudinger, Pointwise estimates and quasilinear parabolic equations, Comm. Pure Appl. Math. 21 (1968), 205-226. MR 37 \#1758.

17. E: D. Williams, The numerical solution of degenerate parabolic equations, Doctoral Dissertation, Texas Tech University, Lubbock, Tex., 1971.

\section{DEPARTMENT OF MATHEMATICS, TEXAS TECH UNIVERSITY, LUBBOCK, TEXAS 79409}

DEPARTMENT OF MATHEMATICS, DISTRICT OF COLUMBI A TEACHERS COLLEGE, WASHINGTON, D. C. 20009 